\title{
Feynman-Hellmann theorem for resonances and the quest for QCD exotica
}

\author{
J. Ruiz de Elvira ${ }^{1, a}{ }_{(\mathbb{D})}$, U.-G. Meißner $^{2,3}$ (D), A. Rusetsky ${ }^{2}$ (D) G. Schierholz ${ }^{4}$ \\ ${ }^{1}$ Albert Einstein Center for Fundamental Physics, Institute for Theoretical Physics, University of Bern, Sidlerstrasse 5, 3012 Bern, Switzerland \\ ${ }^{2}$ Helmholtz-Institut für Strahlen-und Kernphysik and Bethe Center for Theoretical Physics, Universität Bonn, 53115 Bonn, Germany \\ ${ }^{3}$ Institute for Advanced Simulation (IAS-4), Institut für Kernphysik (IKP-3), Jülich Center for Hadron Physics and JARA-HPC, \\ Forschungszentrum Jülich, 52425 Jülich, Germany \\ ${ }^{4}$ Deutsches Elektronen-Synchrotron DESY, 22603 Hamburg, Germany
}

Received: 6 July 2017 / Accepted: 16 September 2017 / Published online: 4 October 2017

(C) The Author(s) 2017. This article is an open access publication

\begin{abstract}
The generalization of the Feynman-Hellmann theorem for resonance states in quantum field theory is derived. On the basis of this theorem, a criterion is proposed to study the possible exotic nature of certain hadronic states emerging in QCD. It is shown that this proposal is supported by explicit calculations in chiral perturbation theory and by large- $N_{c}$ arguments. Analyzing recent lattice data on the quark mass dependence in the pseudoscalar, vector meson, baryon octet and baryon decuplet sectors, we conclude that, as expected, these are predominately quark-model states, albeit the corrections are non-negligible.
\end{abstract}

\section{Introduction}

The celebrated Feynman-Hellmann theorem $[1,2]$ addresses the situation when a quantum-mechanical Hamiltonian $H(\lambda)$ of a given system depends on a some external parameter $\lambda$. The energy spectrum $E_{n}(\lambda)$ and the wave functions $\left|\Psi_{n}(\lambda)\right\rangle$ will then depend on this parameter as well. The theorem relates the $\lambda$-dependence of the energy spectrum to the matrix element of the operator $\mathrm{d} H(\lambda) / \mathrm{d} \lambda$ :

$\frac{\mathrm{d} E_{n}(\lambda)}{\mathrm{d} \lambda}=\left\langle\Psi_{n}(\lambda)\left|\frac{\mathrm{d} H(\lambda)}{\mathrm{d} \lambda}\right| \Psi_{n}(\lambda)\right\rangle$.

In the context of QCD, one often identifies the abstract parameter $\lambda$ with the quark masses $m_{q}$ and studies the dependence of the hadron spectrum on the quark masses. Since the quark-mass-dependent part of the QCD Hamiltonian takes the form $H_{m}=\sum_{q} m_{q} \bar{q} q$, the dependence of, say, the nucleon mass on the quark masses is given by

$\frac{\mathrm{d} m_{N}}{\mathrm{~d} m_{q}}=\frac{1}{2 m_{N}}\langle N|\bar{q} q| N\rangle$.

a e-mail: elvira@itp.unibe.ch
Here, the factor $1 /\left(2 m_{N}\right)$ emerges from the relativistic normalization of the one-particle states, $\left\langle N^{\prime} \mid N\right\rangle=$ $(2 \pi)^{3} 2 E_{N} \delta^{3}\left(\mathbf{p}_{N}^{\prime}-\mathbf{p}_{N}\right)$.

Below, we shall explicitly consider only the three light quark flavors $q=u, d, s$ and, for simplicity, assume that isospin is conserved: $m_{u}=m_{d}=\hat{m}$. The non-strange and strange $\sigma$-terms of the nucleon are defined, respectively, as

$\sigma_{N}=\frac{\hat{m}}{2 m_{N}}\langle N|\bar{u} u+\bar{d} d| N\rangle, \quad \sigma_{N}^{s}=\frac{m_{s}}{2 m_{N}}\langle N|\bar{s} s| N\rangle$,

and the strangeness content of the nucleon is given by

$y=\frac{2\langle N|\bar{s} s| N\rangle}{\langle N|\bar{u} u+\bar{d} d| N\rangle}$.

These $\sigma$-terms contain important information as regards the effect of the explicit chiral symmetry breaking $\left(m_{q} \neq 0\right)$ on the hadronic observables. In addition, the nucleon $\sigma$-term is an important input for the estimates of WIMP cross sections in dark matter direct detection experiments see, e.g., [3-9], as well as in searches for the lepton flavor violation $[10,11]$ and electric dipole moments [12-17]. The strange $\sigma$-term of the nucleon is relevant for the kaon condensation and the formation of the neutron stars, as well as the study of the heavy ion collisions [18-20], etc. The extraction of the $\sigma$-terms from the experimental data is a very delicate issue since, in particular, it implies an analytic continuation of the amplitudes below threshold (to the Cheng-dashed point). In this respect, we note that, from a thorough theoretical analysis of the problem on the basis of dispersion relations and using the input from chiral perturbation theory (ChPT), in Ref. [21] the value $\sigma_{N} \simeq 45 \mathrm{MeV}$ was obtained for the non-strange $\sigma$-term. The most recent and comprehensive analysis, carried out in Ref. [22], is based on the Roy-Steiner equations for $\pi N$ scattering and yields a larger value $\sigma_{N}=(59.1 \pm 3.5) \mathrm{MeV}$. As explained in detail in Ref. [23], most of the difference can 
be traced back to the new and improved values of the pionnucleon scattering lengths deduced from pionic hydrogen and deuterium. This conclusion has been strengthened by a recent reanalysis of the low-energy pion-nucleon scattering data [24].

In recent years, the $\sigma$-terms have also been measured on the lattice [25-31]. In general, two methods are employed in these measurements: a direct measurement of the matrix element and extracting the $\sigma$-terms from the quark mass dependence of the hadron masses with the use of the FeynmanHellmann theorem. The results obtained with the use of both methods are compatible with each other. ${ }^{1}$ The above example is a nice demonstration of the fact that the lattice provides us with additional tools to study the structure of hadrons: while the quark masses on the lattice are free parameters, the experimental data correspond to the physical values of the quark masses, which cannot be varied.

The strangeness content of the nucleon, $y_{N}$, which was defined in Eq. (4), is closely related to the $\sigma$-term and measures the contribution of the strange quarks to the nucleon mass. It can be shown that a large magnitude of the nonstrange $\sigma$-term might signal a large violation of the GellMann-Okubo rule for the baryon octet, and/or a large admixture of the $\bar{s} s$ state to the physical nucleon state that, in its turn, would mean that the properties of the nucleon must differ significantly from those obtained in a simple quark model.

In this paper, we shall demonstrate that the knowledge of the $\sigma$-terms allows one to answer the question as regards the nature of the given hadronic states-namely, whether these states are standard quark-model states ( $q q q$ for baryons, $\bar{q} q$ for mesons), or contain a sizable exotic admixture (pentaquarks, tetraquarks, ...). This criterion, which is a straightforward extension of the argument with the strangeness content of the nucleon, was first proposed in Ref. [33]. In the present article, we extend the discussion to the resonance case. It will be shown that the quark mass dependence of the measured hadron masses in the multiplets obeys certain constraints, if these states are well described by the quark model. Very different constraints emerge, say, for tetraquarks, pentaquarks, etc. We verify this statement by explicit calculations in ChPT, as well as by using large- $N_{c}$ arguments. Note that, at present, a different strategy for identifying the exotic multiquark states is used on the lattice. Namely, one picks up a large set, including both the standard three-quark (quarkantiquark) operators, and the multiquark operators, and calculates the overlap of a given state with the states produced by these operators from the vacuum. A large overlap with the multiquark state would then signal that a given hadron contains a large exotic component and vice versa. Albeit intu-

\footnotetext{
${ }^{1}$ Note that the relation of these extractions for the pion-nucleon $\sigma$-term to the Roy-Steiner analysis is discussed in Ref. [32].
}

itively crystal clear, the argument has to be taken with a grain of salt. The overlap integrals contain information as regards the short-range physics (e.g., about the smearing used in the construction of the operators) and are therefore not observable quantities. Stated differently, all operators which have the same quark content but use different smearing, describe the same state but lead to different overlaps. Consequently, the statements about the nature of the states, which were made on the basis of the calculation of the overlaps, are not completely unambiguous. On the contrary, the measured masses of the hadrons on the lattice are observable quantities and, thus, the constraints on the quark mass dependence, considered in the present article, are devoid of such short-distance ambiguities.

Furthermore, note that all candidates for exotica in QCD are resonances. Consequently, we shall need to extend the derivation of the Feynman-Hellmann theorem to the unstable states in quantum field theory (note that, in Ref. [34], a generalization for the so-called Gamov states has been considered within the framework of non-relativistic quantum mechanics; see also Ref. [35]). This extension is the one of the main results of the present article. Note also that it is not clear, how the overlaps could be used in this case-even in principle. As is well known, resonances do not correspond to a single energy level on the lattice but rather can be associated to a group of a close-by levels. Introducing a novel method to distinguish the exotic states on the lattice seems to be inevitable from this viewpoint as well.

The layout of the present paper is as follows. In Sect. 2, we consider the quark mass dependence in the flavor multiplets and derive the above-mentioned constraints. Section 3 contains the discussion of this dependence within ChPT and large- $N_{c}$ QCD. In Sect. 4, we perform the analysis of the available lattice data from the QCDSF collaboration on the quark mass dependence of the low-lying mesons, baryon octet and baryon decuplet (only stable states) and demonstrate that, as expected, these contain a reasonably small admixture of exotica. Finally, in Sect. 5, the FeynmanHellmann theorem for the resonance states is derived. Section 6 contains our conclusions.

\section{Distinguishing the quark-model states from exotic states}

The very notion of an exotic state in QCD needs the quark model as a reference point. Below, we shall generalize the notion of the strangeness content of the nucleon and define observable quantities, which characterize a given hadronic multiplet. In the quark model, these quantities are given by exact group-theoretical factors. Then the closeness of the measured values of these quantities to the quark-model val- 
ues will be interpreted that the hadrons in this multiplet are predominately non-exotic.

We explain the method for the example of the baryon octet. Let $|B\rangle, B=N, \Sigma, \Xi, \Lambda$, denote the eight different states of the octet. The Gell-Mann-Okubo type relations for the $\bar{q} q$ matrix elements take the form

$$
\begin{aligned}
y_{B} \doteq & \langle B|\bar{s} s| B\rangle=a_{B}+b_{B} Y+c_{B}\left(I(I+1)-\frac{1}{4} Y^{2}-1\right), \\
x_{B} \doteq & \langle B|\bar{u} u+\bar{d} d| B\rangle=a_{B}^{\prime}+b_{B}^{\prime} Y \\
& +c_{B}^{\prime}\left(I(I+1)-\frac{1}{4} Y^{2}-1\right),
\end{aligned}
$$

where $Y$ and $I$ stand, respectively, for the hypercharge and the isospin of the state $|B\rangle$. From the Feynman-Hellmann theorem, one gets

$y_{B}=\frac{\mathrm{d} m_{B}^{2}}{\mathrm{~d} m_{S}}, \quad x_{B}=\frac{\mathrm{d} m_{B}^{2}}{\mathrm{~d} \hat{m}}$.

The following relations are straightforwardly obtained:

$$
\begin{aligned}
& \gamma_{B} \doteq \frac{c_{B}}{a_{B}-c_{B}}=\frac{2 y_{\Sigma}-y_{N}-y_{\Xi}}{2\left(y_{N}+y_{\Xi}\right)-y_{\Sigma}}, \\
& \beta_{B} \doteq \frac{2 b_{B}}{3\left(a_{B}-c_{B}\right)}=\frac{y_{N}-y_{\Xi}}{2\left(y_{N}+y_{\Xi}\right)-y_{\Sigma}}, \\
& \gamma_{B}^{\prime} \doteq \frac{c_{B}^{\prime}}{a_{B}^{\prime}-c_{B}^{\prime}}=\frac{2 x_{\Sigma}-x_{N}-x_{\Xi}}{2\left(x_{N}+x_{\Xi}\right)-x_{\Sigma}}, \\
& \beta_{B}^{\prime} \doteq \frac{2 b_{B}^{\prime}}{3\left(a_{B}^{\prime}-c_{B}^{\prime}\right)}=\frac{x_{N}-x_{\Xi}}{2\left(x_{N}+x_{\Xi}\right)-x_{\Sigma}} .
\end{aligned}
$$

Note that the quantities $\gamma_{B}, \beta_{B}, \gamma_{B}^{\prime}, \beta_{B}^{\prime}$ are scale-invariant in QCD and are therefore devoid of short-range ambiguities.

Further, these ratios can be straightforwardly calculated in the quark model, where the matrix element of the operator $\bar{q} q$ is merely given by the total number of quarks and antiquarks with flavor $f$ present in the state $B$. A simple calculation gives

$\gamma_{B}=\gamma_{B}^{\prime}=0, \quad \beta_{B}=-\frac{2}{3}, \quad \beta_{B}^{\prime}=\frac{1}{3}$.

The same method can be applied, e.g., to the pseudoscalar meson octet. In this case, $\beta_{P}=\beta_{P}^{\prime}=0$ from the beginning, and the remaining coefficients are equal to

$\gamma_{P} \doteq \frac{2\left(y_{\pi}-y_{K}\right)}{4 y_{K}-y_{\pi}}$,

$\gamma_{P}^{\prime} \doteq \frac{2\left(x_{\pi}-x_{K}\right)}{4 x_{K}-x_{\pi}}$,

where

$y_{P}=\frac{\mathrm{d} M_{P}^{2}}{\mathrm{~d} m_{s}}, \quad x_{P}=\frac{\mathrm{d} M_{P}^{2}}{\mathrm{~d} \hat{m}}, \quad P=\pi, K$.

Note that the above relations remain valid even in the presence of $\eta-\eta^{\prime}$ mixing. The matrix elements with $\eta, \eta^{\prime}$ are merely not present there. The quark-model values of the above coefficients are easily calculated:

$\gamma_{P}=-\frac{1}{2}, \quad \gamma_{P}^{\prime}=1$,

whereas, for example, using the wave functions for the tetraquark octet from Ref. [36], one gets completely different values: $\gamma_{P}=1, \gamma_{P}^{\prime}=-\frac{1}{5}$.

The generalization to the other multiplets is straightforward. Below, we present the formulas for the baryon decuplet (note that, using SU(3) symmetry, one may rewrite the following formulas in different equivalent forms):

$\gamma_{\Delta}=\frac{y_{\Omega}-y_{\Sigma^{*}}}{2 y_{\Sigma^{*}}}, \quad \gamma_{\Delta}^{\prime}=\frac{x_{\Omega}-x_{\Sigma^{*}}}{2 x_{\Sigma^{*}}}$.

The quark-model values are $\gamma_{\Delta}=1$ and $\gamma_{\Delta}^{\prime}=-\frac{1}{2}$.

The argument then goes as follows. On the lattice, one may obtain the quark mass dependence of the various members of the multiplets and thus extract the scale-independent quantities $\gamma, \gamma^{\prime}, \beta, \beta^{\prime}$ for a given multiplet. Any statistically significant deviation of these quantities from their quark model values can be interpreted as the effect coming from the SU(3) breaking and/or from the significant admixture of the nonquark-model states. Moreover, on the lattice one could determine the derivatives of the hadron masses with respect to the quark masses in the vicinity of the SU(3)-symmetric point $m_{s}=\hat{m}$ as well. On the basis of this analysis one could then unambiguously judge about the exotic content of the multiplets in the vicinity of this point.

Up to this point, there is little new information. The arguments like given above have been used in the past already (see, e.g., [37]). However, as mentioned in the introduction, one would like to generalize these arguments to the case of unstable states, which do not correspond to a single energy level on the lattice. Note that the different levels may have different quark mass dependence, so the quantities $\gamma, \gamma^{\prime}, \beta, \beta^{\prime}$ cannot be defined unambiguously in this case. However, prior to considering the problem of the resonances, we would like to validate our arguments in the large- $N_{c}$ limit, as well as through direct calculations in ChPT.

\section{Validation of the method}

\subsection{Large- $N_{c}$}

In the context of QCD, the quark-model relations are reproduced in the limit $N_{c} \rightarrow \infty$, which corresponds to the quenching of the virtual quark loops in the path integral. One immediately arrives at the answer in the meson sector. First of all, note that $y_{\pi}=0$ and, consequently, $\gamma_{P}=-\frac{1}{2}$ (without assuming the exact SU(3) symmetry). Further, one gets $x_{\pi}=2 x_{K}$ and the quark-model value $\gamma_{P}^{\prime}=1$ is reproduced. 
The situation with the baryons is more subtle. Of course, in the quenched theory, one immediately gets $y_{N}=0$ (without assuming the exact $\mathrm{SU}(3)$ symmetry) and $y_{\Xi}=2 y_{\Sigma}$, $x_{N}=3 x_{\Xi}, x_{\Sigma}=2 x_{\Xi}$, so that the quark model values $\gamma_{B}=0, \beta_{B}=-\frac{2}{3}, \gamma_{B}^{\prime}=0, \beta_{B}^{\prime}=\frac{1}{3}$ are again reproduced. However, in the large- $N_{c}$ limit, the baryons consist of $N_{c}$ quarks and not just of three quarks-so, the above arguments do not apply straightforwardly. In fact, it was shown that, in this limit, baryons represent static objects (their mass grows like $N_{c}$ ), which at leading order in $N_{c}$ can be described by using, e.g., a constituent quark model [3843]. For any value of $N_{c}$, the baryons, containing $N_{c}$ quarks, belong to a completely symmetric irreducible representation of the SU(6) spin-flavor group. Counterparts of the "usual" baryons $N, \Sigma, \Xi, \ldots$ are those members of the larger multiplets, which have the same spin-flavor quantum numbers. For example, the proton has spin $s=s_{z}=\frac{1}{2}$, isospin $I=I_{z}=\frac{1}{2}$, hypercharge $Y=1$, charge $Q=1$ and baryon number $B=1$ for any given $N_{c}$.

Let us now obtain the values of $\gamma_{B}, \beta_{B}, \gamma_{B}^{\prime}, \beta_{B}^{\prime}$ in the large- $N_{c}$ limit. As known, for an arbitrary $N_{c}$, the generators corresponding to the baryon number and the hypercharge are given by

$\hat{B}=\frac{1}{N_{c}} \operatorname{diag}(1,1,1), \quad \hat{Y}=\frac{1}{N_{c}} \operatorname{diag}\left(1,1,1-N_{c}\right)$.

It is straightforward to check that the mass term in the Lagrangian is given by

$\mathcal{L}_{m}=\hat{m} \bar{\psi}\left(\left(N_{c}-1\right) \hat{B}+\hat{Y}\right) \psi+m_{s} \bar{\psi}(\hat{B}-\hat{Y}) \psi$.

From the above equation one may read off the values for $x_{B}, y_{B}$ (up to an overall normalization factor)

$x_{N}=\left(N_{c}-1\right)+1, \quad x_{\Sigma}=\left(N_{c}-1\right)$,

$x_{\Xi}=\left(N_{c}-1\right)-1, \quad y_{N}=0, \quad y_{\Sigma}=1, \quad y_{\Xi}=2$,

and, finally,

$\gamma_{B}=\gamma_{B}^{\prime}=0, \quad \beta_{B}=-\frac{2}{3}, \quad \beta_{B}^{\prime}=\frac{2}{3\left(N_{c}-1\right)}$.

As we see, the quantity $\beta_{B}$ stays finite in the large- $N_{c}$ limit, whereas the quantities $\gamma_{B}, \gamma_{B}^{\prime}, \beta_{B}^{\prime}$ all vanish in this limit. On the other hand, at $N_{c}=3$ the quark model results are reproduced. Note also that our results are in complete agreement with Ref. [44] at the leading order in $1 / N_{c}$.

\subsection{Chiral perturbation theory}

\subsubsection{Goldstone boson octet}

At one loop, the pion and kaon masses in 3-flavor ChPT are given by (see, e.g. [45])

$$
\begin{aligned}
& M_{\pi}^{2}=\stackrel{\circ}{M}_{\pi}^{2}\left\{1+\mu_{\pi}-\frac{1}{3} \mu_{\eta}+2 \hat{m} K_{3}+K_{4}\right\} \\
& M_{K}^{2}=\stackrel{\circ}{M}_{K}^{2}\left\{1+\frac{2}{3} \mu_{\eta}+\left(\hat{m}+m_{s}\right) K_{3}+K_{4}\right\},
\end{aligned}
$$

where

$\mu_{P}=\frac{\stackrel{\circ}{M}_{P}^{2}}{32 \pi^{2} F_{0}^{2}} \ln \frac{\stackrel{\circ}{M}_{P}^{2}}{\mu^{2}}, \quad P=\pi, K, \eta$,

$\stackrel{\circ}{M}_{\pi}^{2}=2 \hat{m} B_{0}, \quad \stackrel{\circ}{M}_{K}^{2}=\left(\hat{m}+m_{s}\right) B_{0}$,

$\stackrel{\circ}{M}_{\eta}^{2}=\frac{2}{3}\left(\hat{m}+2 m_{s}\right) B_{0}, \quad K_{3}=\frac{8 B_{0}}{F_{0}^{2}}\left(2 L_{8}^{r}-L_{5}^{r}\right)$,

$K_{4}=\left(2 \hat{m}+m_{s}\right) \frac{16 B_{0}}{F_{0}^{2}}\left(2 L_{6}^{r}-L_{4}^{r}\right)$,

with $B_{0}, F_{0}, L_{i}^{r}$ the parameters of the ChPT Lagrangian, $\stackrel{\circ}{M}_{P}^{2}$ are the pseudoscalar meson masses squared at leading order, and $\mu$ denotes the scale of dimensional regularization. Calculating the parameters $\gamma_{P}, \gamma_{P}^{\prime}$ from these expressions, we get

$$
\begin{aligned}
& \gamma_{P}=-\frac{1}{2}\left\{1+\frac{\stackrel{\circ}{M}_{\pi}^{2}}{96 \pi^{2} F_{0}^{2}}\left(\ln \frac{\stackrel{\circ}{M}_{\eta}^{2}}{\mu^{2}}+1\right)\right. \\
& \left.-\frac{12 \stackrel{\circ}{M}_{\pi}^{2}}{F_{0}^{2}}\left(2 L_{6}^{r}-L_{4}^{r}\right)\right\} \text {, } \\
& \gamma_{P}^{\prime}=1+\frac{3 \stackrel{\circ}{M}_{\pi}^{2}}{16 \pi^{2} F_{0}^{2}} \ln \frac{\stackrel{\circ}{M}_{\pi}^{2}}{\mu^{2}}-\frac{6 \stackrel{\circ}{M}_{\eta}^{2}+\stackrel{\circ}{M}_{\pi}^{2}}{48 \pi^{2} F_{0}^{2}} \ln \frac{\stackrel{\circ}{M}_{\eta}^{2}}{\mu^{2}} \\
& +\frac{7 \stackrel{\circ}{M}_{\pi}^{2}-3 \stackrel{\circ}{M}_{\eta}^{2}}{96 \pi^{2} F_{0}^{2}}+\frac{36\left(\stackrel{\circ}{M}_{\pi}^{2}-\stackrel{\circ}{M}_{\eta}^{2}\right)}{F_{0}^{2}}\left(2 L_{8}^{r}-L_{5}^{r}\right) \\
& -\frac{24\left(3 \stackrel{\circ}{M}_{\eta}^{2}-\stackrel{\circ}{M}_{\pi}^{2}\right)}{F_{0}^{2}}\left(2 L_{6}^{r}-L_{4}^{r}\right) \text {. }
\end{aligned}
$$

If we recall now that, in the large- $N_{c}$ limit, $F_{0}=O\left(N_{c}^{1 / 2}\right)$, $L_{5}^{r}, L_{8}^{r}=O\left(N_{c}\right), L_{4}^{r}, L_{6}^{r}=O(1)$ and the meson masses are of order 1 as well, it is seen that $\gamma_{P}, \gamma_{P}^{\prime}$ tend to the quarkmodel values in the limit $N_{c} \rightarrow \infty$ and $m_{s}=\hat{m}$. Further, one could estimate the deviation of these parameters in the real world from their exact quark-model values. To this end, we replace $F_{0}$ by the pion decay constant $F_{\pi}=92.2 \mathrm{MeV}$ and $\circ$
$M_{\pi}, \stackrel{\circ}{M}_{K}$ by the physical meson masses $\left(\stackrel{\circ}{M_{\eta}}\right.$ is determined from the Gell-Mann-Okubo relation). Using the central val- 
ues for the low-energy constants (LECs) $L_{i}^{r}$ from Ref. [45] at $\mu=770 \mathrm{MeV}$

$L_{4}^{r}=L_{6}^{r}=0, \quad L_{5}^{r}=2.2 \cdot 10^{-3}, \quad L_{8}^{r}=1.1 \cdot 10^{-3}$,

we get

$\gamma_{P}=-\frac{1}{2}\left(1+9 \cdot 10^{-4}\right), \quad \gamma_{P}^{\prime}=1+4 \cdot 10^{-2}$.

In order to estimate the uncertainty, we present the results obtained by using the LECs from Ref. [46]. There are different sets of LECs in this paper. We use the $O\left(p^{4}\right)$ fit from Table 1: $L_{4}^{r}=L_{6}^{r}=0, L_{5}^{r}=1.2 \times 10^{-3}, L_{8}^{r}=0.5 \times 10^{-3}$, as well as the fit to lattice data: $L_{4}^{r}=0.04 \times 10^{-3}, L_{6}^{r}=$ $0.07 \times 10^{-3}, L_{5}^{r}=0.84 \times 10^{-3}, L_{8}^{r}=0.36 \times 10^{-3}$; see Table 5 of this paper. The results are

$\begin{array}{ll}\gamma_{P}=-\frac{1}{2}\left(1+9 \times 10^{-4}\right), & \gamma_{P}^{\prime}=1+0.30 \quad\left[O\left(p^{4}\right)\right], \\ \gamma_{P}=-\frac{1}{2}\left(1-2 \times 10^{-3}\right), & \gamma_{P}^{\prime}=1-7 \times 10^{-2} \quad \text { [lattice], }\end{array}$

The SU(3)-symmetric point is achieved from the physical point by varying the quark masses such that the sum of all quark masses $2 \hat{m}+m_{s}=$ const. This corresponds to $M_{\pi}=$ $M_{K}=M_{\eta}=413 \mathrm{MeV}$. At this point, using the LECs from Ref. [45], we get

$\gamma_{P}=-\frac{1}{2}\left(1-5 \times 10^{-3}\right), \quad \gamma_{P}^{\prime}=1-2 \times 10^{-2}$,

whereas the use of the LECs from Ref. [46] gives the following answer:

$\gamma_{P}=-\frac{1}{2}\left(1-5 \times 10^{-3}\right), \quad \gamma_{P}^{\prime}=1-2 \times 10^{-2}\left[O\left(p^{4}\right)\right]$,

$\gamma_{P}=-\frac{1}{2}\left(1-3 \times 10^{-2}\right), \quad \gamma_{P}^{\prime}=1-0.12 \quad$ [lattice].

As we see, the corrections to the quark model values for the pseudoscalar octet are reasonably small. The uncertainties, which stem from the LECs, are, however, sizable and the correlations between various LECs should be taken into account.

\subsubsection{Ground-state baryon octet}

The case of the baryon octet is another illustration of the method. One could use the result on the baryon masses in ChPT, which are available in the literature; see e.g. Refs. [4749]. In this article, we restrict ourselves to the $O\left(p^{2}\right)$ calculations. The relevant part of the effective Lagrangian is given by

$$
\begin{aligned}
\mathcal{L}^{(2)}= & b_{D}\left\langle\bar{B}\left\{\chi_{+}, B\right\}\right\rangle+b_{F}\left\langle\bar{B}\left[\chi_{+}, B\right]\right\rangle \\
& +b_{0}\langle\bar{B} B\rangle\left\langle\chi_{+}\right\rangle+\cdots,
\end{aligned}
$$

where the matrix $B$ is the baryon octet field and $\chi_{+}=$ $u^{\dagger} \chi u^{\dagger}+u \chi u$, with $\chi=2 B_{0} \mathcal{M}, \mathcal{M}=\operatorname{diag}\left(\hat{m}, \hat{m}, m_{s}\right)$. Further, $u=\exp \left(i \phi /\left(2 F_{0}\right)\right)$ is the Goldstone boson field and $b_{D}, b_{F}, b_{0}$ are the pertinent LECs. The expressions for the coefficients $\gamma_{B}, \gamma_{B}^{\prime}, \beta_{B}, \beta_{B}^{\prime}$ can be readily obtained from the calculated baryon masses at this order

$\gamma_{B}=-\frac{2 b_{D}}{3 b_{0}+4 b_{D}}, \quad \gamma_{B}^{\prime}=\frac{b_{D}}{3 b_{0}+b_{D}}$,

$\beta_{B}=-\frac{2 b_{F}}{3 b_{0}+4 b_{D}}, \quad \beta_{B}^{\prime}=\frac{b_{F}}{3 b_{0}+b_{D}}$.

Unlike the Goldstone boson case, these expressions do not reduce automatically at lowest order to the quark-model values given by Eq. (8). The quark-model results are reproduced, if $b_{D} / b_{0}=0$ and $b_{F} / b_{0}=1$. This statement seems to be supported by phenomenological values of these LECs [47] (all in $\mathrm{GeV}^{-1}$ units)

$-0.79 \leq b_{0} \leq-0.70, \quad 0.01 \leq b_{D} \leq 0.07$,

$-0.61 \leq b_{F} \leq-0.48$.

It could be interesting to consider the constraints on the higher-order LECs, which emerge in a similar fashion and, possibly, from the observables other than the baryon masses as well. However, since the convergence in the SU(3) baryon ChPT is a somewhat painful issue [50,51], we do not consider any further constraints here. The results of Ref. [52,53] support this statement. For example, the NLO results for these constants are $b_{0}=-0.273(6), b_{D}=$ 0.0506(17), $b_{F}=-0.179(1)$, whereas at the NNLO one has $b_{0}=-0.886(5), b_{D}=0.0482(17), b_{F}=-0.517(7)$ (all in $\mathrm{GeV}^{-1}$ units). One sees that the general pattern persists, albeit the values of the LECs vary quite a bit.

In Ref. [44], comparing the chiral Lagrangians at arbitrary $N_{c}$ and at $N_{c}=3$, it was shown that $b_{D} / b_{0}=0$ and $b_{F} / b_{0}=$ 1 hold in the limit of large $N_{c}$. Therefore, the quark-model values are exact at $N_{c}=3$ up to the corrections that vanish in the large- $N_{c}$ limit. Note also that in Eq. (26) the large$N_{c}$ limit cannot be performed straightforwardly, because it is obtained from the Lagrangian given in Eq. (25), which is defined at $N_{c}=3$ only. In fact, as easily seen, the above constraints on $b_{0}, b_{D}, b_{F}$ are incompatible with $\beta_{B}^{\prime} \rightarrow 0$ and $\beta_{B} \neq 0$ in the large- $N_{c}$ limit.

\subsubsection{Ground-state vector meson octet}

The vector meson octet contains unstable particles. So, strictly speaking, our formulas are not directly applicable there. However, in order to get an intuitive understanding of the problem, one could still use an effective Lagrangian with vector mesons (see, e.g., Ref. [54]) and evaluate the pertinent coefficients $\gamma_{V}, \gamma_{V}^{\prime}$, defined through the Eqs. $(9,10)$ with the replacements $\pi \rightarrow \rho$ and $K \rightarrow K^{*}$. The quark-mass- 
dependent part of the effective Lagrangian is given by [54]

$$
\begin{aligned}
\mathcal{L}_{m}= & a_{1}\left\langle\left\{W_{\mu}^{\dagger}, W^{\mu}\right\} \chi_{+}\right\rangle+a_{13}\left\langle W_{\mu}^{\dagger} W^{\mu}\right\rangle\left\langle\chi_{+}\right\rangle \\
& +c_{1}\left\langle W_{\mu}^{\dagger} \chi_{+} W^{\mu} \chi_{+}\right\rangle+c_{2}\left\langle\left\{W_{\mu}^{\dagger}, W^{\mu}\right\} \chi_{+} \chi_{+}\right\rangle,
\end{aligned}
$$

where the LEC $a_{13}$ is suppressed at $O\left(N_{c}^{-1}\right)$ with respect to the other LECs (we do not display the $1 / N_{c}$-suppressed terms at order $p^{4}$ ).

As is well known, the Goldstone boson loops are suppressed by a factor $N_{c}$. For this reason, we drop them in the expression of the masses completely. The contact term contribution to the masses is given by

$$
\begin{aligned}
M_{\rho}^{2}= & \stackrel{\circ}{M}_{V}^{2}+8 a_{1} B_{0} \hat{m}+4 a_{13} B_{0}\left(2 \hat{m}+m_{s}\right) \\
& +16\left(c_{1}+2 c_{2}\right)\left(B_{0} \hat{m}\right)^{2}, \\
M_{K^{*}}^{2}= & \stackrel{\circ}{M}_{V}^{2}+4 a_{1} B_{0}\left(\hat{m}+m_{s}\right)+4 a_{13} B_{0}\left(2 \hat{m}+m_{s}\right) \\
& +16 c_{1} B_{0}^{2} \hat{m} m_{s}+16 c_{2} B_{0}^{2}\left(\hat{m}^{2}+\hat{m}_{s}^{2}\right),
\end{aligned}
$$

with $\stackrel{\circ}{M}_{V}^{2}$ the vector meson octet mass in the chiral limit. From this, one can immediately read off the expressions for $\gamma_{V}, \gamma_{V}^{\prime}:$

$$
\begin{aligned}
\gamma_{V}= & \frac{2\left(y_{\rho}-y_{K^{*}}\right)}{4 y_{K^{*}}-y_{\rho}}=-\frac{1}{2} \frac{4 a_{1}}{4 a_{1}+3 a_{13}} \\
& +O\left(p^{4}\right)+O\left(p^{2} N_{c}^{-1}\right), \\
\gamma_{V}^{\prime}= & \frac{2\left(x_{\rho}-x_{K^{*}}\right)}{4 x_{K^{*}}-x_{\rho}}=\frac{a_{1}}{a_{1}+3 a_{13}}-\frac{12 c_{1}\left(M_{K}^{2}-M_{\pi}^{2}\right)}{a_{1}+a_{13}} \\
& +O\left(p^{4}\right)+O\left(p^{2} N_{c}^{-1}\right) .
\end{aligned}
$$

It is seen that the corrections to $\gamma_{V}, \gamma_{V}^{\prime}$ vanish in the large- $N_{c}$ and $\mathrm{SU}(3)$ symmetry limit. From this example, it becomes clear, how the effective theory for the exotic multiplets (e.g., the scalar mesons) could be constructed. The operator basis, whose form is dictated by the symmetries, is the same in the case of the standard and exotic particles. The difference emerges at the level of the LECs: certain LECs in the effective Lagrangian with exotic particles are not suppressed in the large- $N_{c}$ limit.

\section{Testing with lattice data}

The QCDSF collaboration has studied the quark mass dependence of hadron masses in various meson and baryon multiplets. This analysis provides us with an ideal input to test our predictions. In order to make the comparison straightforward, both for the bare and renormalized quark masses, we define

$$
\begin{aligned}
\bar{m} & =\frac{1}{3}\left(2 \hat{m}+m_{s}\right), \quad m_{1}=\hat{m}-m_{s}, \\
\bar{m}^{r} & =\frac{1}{3}\left(2 \hat{m}^{r}+m_{s}^{r}\right), \quad m_{1}^{r}=\hat{m}^{r}-m_{s}^{r},
\end{aligned}
$$

The bare and renormalized quark masses are related by

$\bar{m}^{r}=Z_{m}^{S} \bar{m}, \quad m_{1}^{r}=Z_{m}^{N S} m_{1}, \quad Z_{m}^{S} / Z_{m}^{N S}=1+\alpha_{Z}$.

Calculating the derivatives with respect to the renormalized masses, we get

$$
\begin{aligned}
& \left.y_{a} \doteq \frac{\partial M_{a}^{2}}{\partial m_{s}^{r}}\right|_{\hat{m}^{r}=\mathrm{const}}=\left.\frac{1}{3} \frac{\partial M_{a}^{2}}{\partial \bar{m}^{r}}\right|_{m_{1}^{r}=\mathrm{const}}-\left.\frac{\partial M_{a}^{2}}{\partial m_{1}^{r}}\right|_{\bar{m}^{r}=\mathrm{const}}, \\
& \left.x_{a} \doteq \frac{\partial M_{a}^{2}}{\partial \hat{m}^{r}}\right|_{m_{s}^{r}=\mathrm{const}}=\left.\frac{2}{3} \frac{\partial M_{a}^{2}}{\partial \bar{m}^{r}}\right|_{m_{1}^{r}=\mathrm{const}}+\left.\frac{\partial M_{a}^{2}}{\partial m_{1}^{r}}\right|_{\bar{m}^{r}=\mathrm{const}} .
\end{aligned}
$$

Here, the $M_{a}$ denote the hadron masses in a given multiplet. One may relate this to the derivative with respect to the bare masses

$$
\begin{aligned}
& \frac{\partial M_{a}^{2}}{\partial \bar{m}^{r}}=\frac{1}{Z_{m}^{S}} \frac{\partial M_{a}^{2}}{\partial \bar{m}}=\frac{1}{Z_{m}^{N S}\left(1+\alpha_{Z}\right)} \frac{\partial M_{a}^{2}}{\partial \bar{m}}, \\
& \frac{\partial M_{a}^{2}}{\partial \bar{m}_{1}^{r}}=\frac{1}{Z_{m}^{N S}} \frac{\partial M_{a}^{2}}{\partial m_{1}} .
\end{aligned}
$$

SU(3) symmetry in the vicinity of the symmetric point introduces additional constraints on the derivatives with respect to $m_{1}$. Namely, retaining only linear terms in $m_{1}$, the hadron masses in the multiplets are given by [55]

pseudoscalar octet:

$M_{\pi}^{2}=M_{P}^{2}+\frac{2}{3} \alpha_{P} m_{1}, \quad M_{K}^{2}=M_{P}^{2}-\frac{1}{3} \alpha_{P} m_{1}$,

$M_{\eta_{s}}^{2}=M_{P}^{2}-\frac{4}{3} \alpha_{P} m_{1}$.

vector octet:

$$
\begin{aligned}
M_{\rho} & =M_{V}+\frac{2}{3} \alpha_{V} m_{1}, \quad M_{K^{*}}=M_{V}-\frac{1}{3} \alpha_{V} m_{1}, \\
M_{\phi_{s}} & =M_{V}-\frac{4}{3} \alpha_{V} m_{1} .
\end{aligned}
$$

baryon octet:

$$
\begin{array}{ll}
M_{N}=M_{B}+A_{1} m_{1}, & M_{\Lambda}=M_{B}+A_{2} m_{1}, \\
M_{\Sigma}=M_{B}-A_{2} m_{1}, & M_{\Xi}=M_{B}-\left(A_{1}-A_{2}\right) m_{1} .
\end{array}
$$

baryon decuplet:

$$
\begin{aligned}
M_{\Delta} & =M_{D}+A m_{1}, \quad M_{\Sigma^{*}}=M_{D}, \\
M_{\Xi^{*}} & =M_{D}-A m_{1}, \quad M_{\Omega}=M_{D}-2 m_{1} .
\end{aligned}
$$

The expansion coefficients depend on the variable $\bar{m}$. Calculating the derivatives with respect to the variables $\bar{m}, m_{1}$ and introducing the notation

$$
\begin{aligned}
\lambda_{P} & =\frac{1}{1+\alpha_{Z}} \frac{d M_{P}^{2}}{d \bar{m}}, \quad \lambda_{A}=\frac{1}{1+\alpha_{Z}} \frac{d M_{A}}{d \bar{m}}, \\
A & =V, B, D
\end{aligned}
$$


one gets

$$
\begin{aligned}
& \gamma_{P}=\frac{-2\left(\alpha_{P} / \lambda_{P}\right)}{1+2\left(\alpha_{P} / \lambda_{P}\right)}, \quad \gamma_{P}^{\prime}=\frac{\left(\alpha_{P} / \lambda_{P}\right)}{1-\left(\alpha_{P} / \lambda_{P}\right)}, \\
& \gamma_{V}=\frac{-2\left(\alpha_{V} / \lambda_{V}\right)}{1+2\left(\alpha_{V} / \lambda_{V}\right)}, \quad \gamma_{V}^{\prime}=\frac{\left(\alpha_{V} / \lambda_{V}\right)}{1-\left(\alpha_{V} / \lambda_{V}\right)}, \\
& \gamma_{B}=\frac{\left(3 A_{2} / \lambda_{B}\right)}{1-\left(3 A_{2} / \lambda_{B}\right)}, \quad \gamma_{B}^{\prime}=\frac{-\left(3 A_{2} / \lambda_{B}\right) / 2}{1-\left(3 A_{2} / \lambda_{B}\right) / 2}, \\
& \beta_{B}=\frac{-2 A_{1} / \lambda_{B}+A_{2} / \lambda_{B}}{1-\left(3 A_{2} / \lambda_{B}\right)}, \quad \beta_{B}^{\prime}=\frac{A_{1} / \lambda_{B}-A_{2} /\left(2 \lambda_{B}\right)}{1+\left(3 A_{2} / \lambda_{B}\right) / 2}, \\
& \gamma_{D}=\frac{3 A}{\lambda_{D}}, \quad \gamma_{D}^{\prime}=-\frac{3 A}{2 \lambda_{D}} .
\end{aligned}
$$

Note that the above relations are valid exactly in the SU(3)symmetric point, where $m_{1}=0$ and the masses of all hadrons in the same multiplet are equal.

The quark-model values are

$$
\begin{aligned}
& \frac{2 \alpha_{P}}{\lambda_{P}}=\frac{2 \alpha_{V}}{\lambda_{V}}=1, \quad \frac{3 A_{1}}{\lambda_{B}}=1, \\
& \frac{3 A_{2}}{\lambda_{B}}=0, \quad \frac{3 A}{\lambda_{D}}=1,
\end{aligned}
$$

whereas, e.g., the tetraquark value is completely different:

$\frac{2 \alpha_{T}}{\lambda_{T}}=-\frac{1}{2}$.

That is, even the sign is different from the case of the the ordinary $\bar{q} q$-mesons, allowing one to clearly distinguish between both QCD configurations.

Having set the definitions, we proceed with the verification of our method using the lattice data from Ref. [55]. We will only consider results corresponding to the largest lattice size $32^{3} \times 64$, hence avoiding the discussion of finite-size lattice corrections. Bare quark masses are defined as

$a m_{q}=\frac{1}{2}\left(\frac{1}{\kappa_{q}}-\frac{1}{\kappa_{0, c}}\right)$.

On the symmetric line, i.e. for $\hat{m}=m_{s}=\bar{m}$ and $\kappa_{l}=$ $\kappa_{s}=\kappa_{0}$, the QCDSF results for the pseudoscalars, vectors, octet and decuplet baryons are given in Table 1 . Note that the results corresponding to the second row, $\kappa_{0}=0.12092$, have been slightly updated with respect to the values in Ref. [55].

Table 1 Updated values of hadron masses on the symmetric line from the $32^{3} \times 64$ lattice [55]. The input in the second row is updated as compared to Ref. [55] (this is marked by the $*$ )

\begin{tabular}{lllll}
\hline$\kappa_{0}$ & $a M_{\pi}$ & $a M_{\rho}$ & $a M_{N}$ & $a M_{\Delta}$ \\
\hline 0.12090 & $0.1747(5)$ & $0.3341(34)$ & $0.4673(27)$ & $0.5675(64)$ \\
$0.12092 *$ & $0.1647(4)$ & $0.3282(41)$ & $0.4443(59)$ & $0.5577(112)$ \\
0.12095 & $0.1508(4)$ & $0.3209(27)$ & $0.4329(49)$ & $0.5541(80)$ \\
0.12099 & $0.1285(7)$ & $0.3006(59)$ & $0.4107(89)$ & $0.5183(157)$ \\
\hline
\end{tabular}

Chiral symmetry requires the pion mass to vanish in the chiral $\bar{m} \rightarrow 0$ limit, which allows one to determine the critical hopping parameter $\kappa_{0, c}$ by extrapolating the pseudoscalar masses through the symmetric line. Thus, considering at first order

$M_{\pi}^{2}=\frac{a_{\pi}}{\kappa_{0}}+b_{\pi}=a_{\pi}\left(\frac{1}{\kappa_{0}}-\frac{1}{\kappa_{0, c}}\right)=2 a_{\pi} \bar{m}, \kappa_{0, c}=-\frac{a_{\pi}}{b_{\pi}}$,

and fitting the data in Table 1, one can directly extract the value of the $a_{\pi}$ and $b_{\pi}$ coefficients, namely

$a_{\pi}=\frac{1}{2} \frac{d M_{P}^{2}}{d \bar{m}}=2.249 \pm 0.037, \quad b_{\pi}=-18.57 \pm 0.31$,

so that the critical hopping parameter reads

$\kappa_{0, c}=0.12098 \pm 0.00283$.

The uncertainties have been computed using a bootstrap with a normally distributed sample of 1000 points, with a standard deviation defined from the $68 \%$ of the distribution.

Once the critical hopping parameter $\kappa_{0, c}$ has been obtained, Eq. (43) allows one to study the behavior of the vector and octet and decuplet baryons at the symmetric line. Considering again a linear fit to the data in Table 1, we obtain $\frac{\mathrm{d} M_{V}}{\mathrm{~d} \bar{m}}=7.66 \pm 2.47, \quad \frac{\mathrm{d} M_{B}}{\mathrm{~d} \bar{m}}=19.33 \pm 2.3$, $\frac{\mathrm{d} M_{D}}{\mathrm{~d} \bar{m}}=12.28 \pm 4.13$

where the errors have been computed using again a bootstrap method.

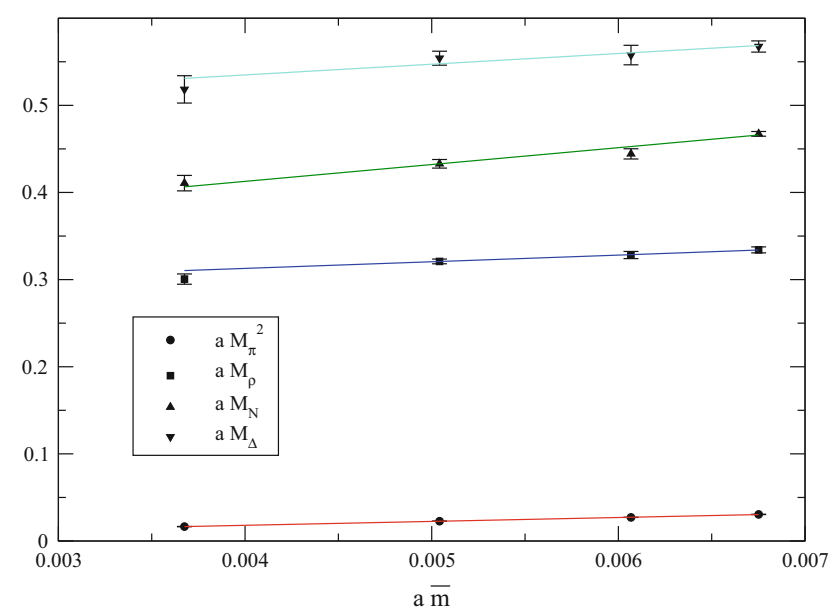

Fig. 1 Linear fit for pseudoscalar and vector mesons and octet and decuplet baryon along the symmetric line. In the case of the vectors, only the first three points have been considered in the fit, since the last one corresponds to an unstable particle 
Table 2 Pseudoscalar meson masses $a M_{\pi}, a M_{K}$ and $a M_{\eta_{s}}$ and vector meson masses $M_{\rho}$, $a M_{K^{*}}$ and $a M_{\phi_{s}}$ on the $\bar{m}=$ constant line from the $32^{3} \times 64$ lattice [55]. The input in the fourth row is updated as compared to Ref. [55] (this is marked by the $*$ )

\begin{tabular}{llll}
\hline$\left(\kappa_{l}, \kappa_{s}\right)$ & $a M_{\pi}$ & $a M_{K}$ & $a M_{\eta_{s}}$ \\
\hline$(0.120900,0.120900)$ & $0.1747(5)$ & $0.1747(5)$ & $0.1747(5)$ \\
$(0.121040,0.120620)$ & $0.1349(5)$ & $0.1897(4)$ & $0.2321(3)$ \\
$(0.121095,0.120512)$ & $0.1162(8)$ & $0.1956(5)$ & $0.2512(3)$ \\
$(0.121145,0.120413) *$ & $0.09687(84)$ & $0.2015(4)$ & $0.2682(3)$ \\
\hline$\left(\kappa_{l}, \kappa_{s}\right)$ & $a M_{\rho}$ & $a M_{K^{*}}$ & $a M_{\phi_{s}}$ \\
\hline$(0.120900,0.120900)$ & $0.3341(34)$ & $0.3341(34)$ & $0.3341(34)$ \\
$(0.121040,0.120620)$ & $0.3127(38)$ & $0.3380(21)$ & $0.3632(14)$ \\
$(0.121095,0.120512)$ & $0.3123(43)$ & $0.3426(20)$ & $0.3738(11)$ \\
$(0.121145,0.120413) *$ & $0.3227(65)$ & $0.3490(22)$ & $0.3874(11)$
\end{tabular}

Table 3 Octet baryon masses $a M_{N}, a M_{\Lambda}, a M_{\Sigma}$ and $a M_{\Xi}$ and decuplet baryon masses $M_{\Delta}$, $a M_{\Sigma^{*}}, a M_{\Xi^{*}}$ and $a M_{\Omega}$ on the $\bar{m}=$ constant line for the $32^{3} \times 64$ lattice [55]. The input in the fourth row is updated as compared to Ref. [55] (this is marked by the $*$ )

\begin{tabular}{lllll}
\hline$\left(\kappa_{l}, \kappa_{s}\right)$ & $a M_{N}$ & $a M_{\Lambda}$ & $a M_{\Sigma}$ & $a M_{\Xi}$ \\
\hline$(0.120900,0.120900)$ & $0.4673(27)$ & $0.4673(27)$ & $0.4673(27)$ & $0.4673(27)$ \\
$(0.121040,0.120620)$ & $0.4267(50)$ & $0.4547(43)$ & $0.4697(33)$ & $0.4907(21)$ \\
$(0.121095,0.120512)$ & $0.4140(61)$ & $0.4510(58)$ & $0.4690(37)$ & $0.4971(21)$ \\
$(0.121145,0.120413) *$ & $0.3950(114)$ & $0.4460(65)$ & $0.4739(42)$ & $0.5073(21)$ \\
\hline$\left(\kappa_{l}, \kappa_{s}\right)$ & $a M_{\Delta}$ & $a M_{\Sigma^{*}}$ & $a M_{\Xi^{*}}$ & $a M_{\Omega}$ \\
\hline$(0.120900,0.120900)$ & $0.5675(64))$ & $0.5675(64)$ & $0.5675(64)$ & $0.5675(64)$ \\
$(0.121040,0.120620)$ & $0.5520(79)$ & $0.5744(48)$ & $0.5968(34)$ & $0.6194(28)$ \\
$(0.121095,0.120512)$ & $0.5161(185)$ & $0.5541(98)$ & $0.5812(52)$ & $0.6104(33)$ \\
$(0.121145,0.120413) *$ & $0.4808(320)$ & $0.5441(136)$ & $0.5956(68)$ & $0.6382(35)$ \\
\hline
\end{tabular}

The fit results, together with the lattice data in Table 1 are plotted in Fig. 1, showing a nice linear behavior and hence the fit quality. Note that for the vector mesons we have got rid of the last data point, which lies quite well above the $2 m_{\pi}$ threshold and hence corresponds to an unstable particle.

Beyond the symmetric line, no reference to $\kappa_{0, c}$ is needed, since

$a m_{1}=\frac{1}{2}\left(\frac{1}{\kappa_{l}}-\frac{1}{\kappa_{s}}\right)$.

The QCDSF collaboration results for pseudoscalar and vector mesons, and octet and decuplet baryons corresponding to the $32^{3} \times 64$ lattice size can be found in Tables 2 and 3 . Once more, the results of the fourth row, i.e. for $\kappa_{l}=0.121145$ and $\kappa_{s}=0.120413$, are also updated as compared with Ref. [55].

Using these data together to Eq. (48), we can perform a linear fit to compute the coefficients in Eqs. (36)-(38), getting

$$
\begin{aligned}
\alpha_{P} & =1.242 \pm 0.003, \quad \alpha_{V}=1.64 \pm 0.05, \\
A_{1} & =2.26 \pm 0.09, \quad A_{2}=0.51 \pm 0.05 \\
A & =1.33 \pm 0.07,
\end{aligned}
$$

where, once more, the errors have been computed using a bootstrap with a normally distributed sample of 1000 points, and the fit results have been plotted in Fig. 2. As in the symmetric case, we have not included the last two points for the $\rho$ and $K^{*}$ mesons, which correspond to mass values well above the $\pi \pi$ and $\pi K$ threshold, respectively, and hence to unstable particles. As one can see in Fig. 2, the linear fits work extremely well for pseudoscalar mesons, and relatively well for vectors and octet baryons. On the contrary, the decuplet results show deviations from a linear behavior. Nevertheless, their description does not improve with a quadratic function either and a linear behavior will be assumed in order to test our criterion for the decuplet.

\section{Pseudoscalar mesons}

Since the data on the pseudoscalar meson masses are almost linear in the quark masses-in other words, the Gell-MannOakes-Renner relation is obeyed very well, we will assume an exact linear dependence to extract the renormalization constant $\alpha_{Z}$,

$\alpha_{Z}=\frac{1}{2 \alpha_{P}} \frac{\mathrm{d} M_{P}^{2}}{\mathrm{~d} \bar{m}}-1=0.811 \pm 0.029$,

so our criterion turns into an identity for the pseudoscalar mesons 

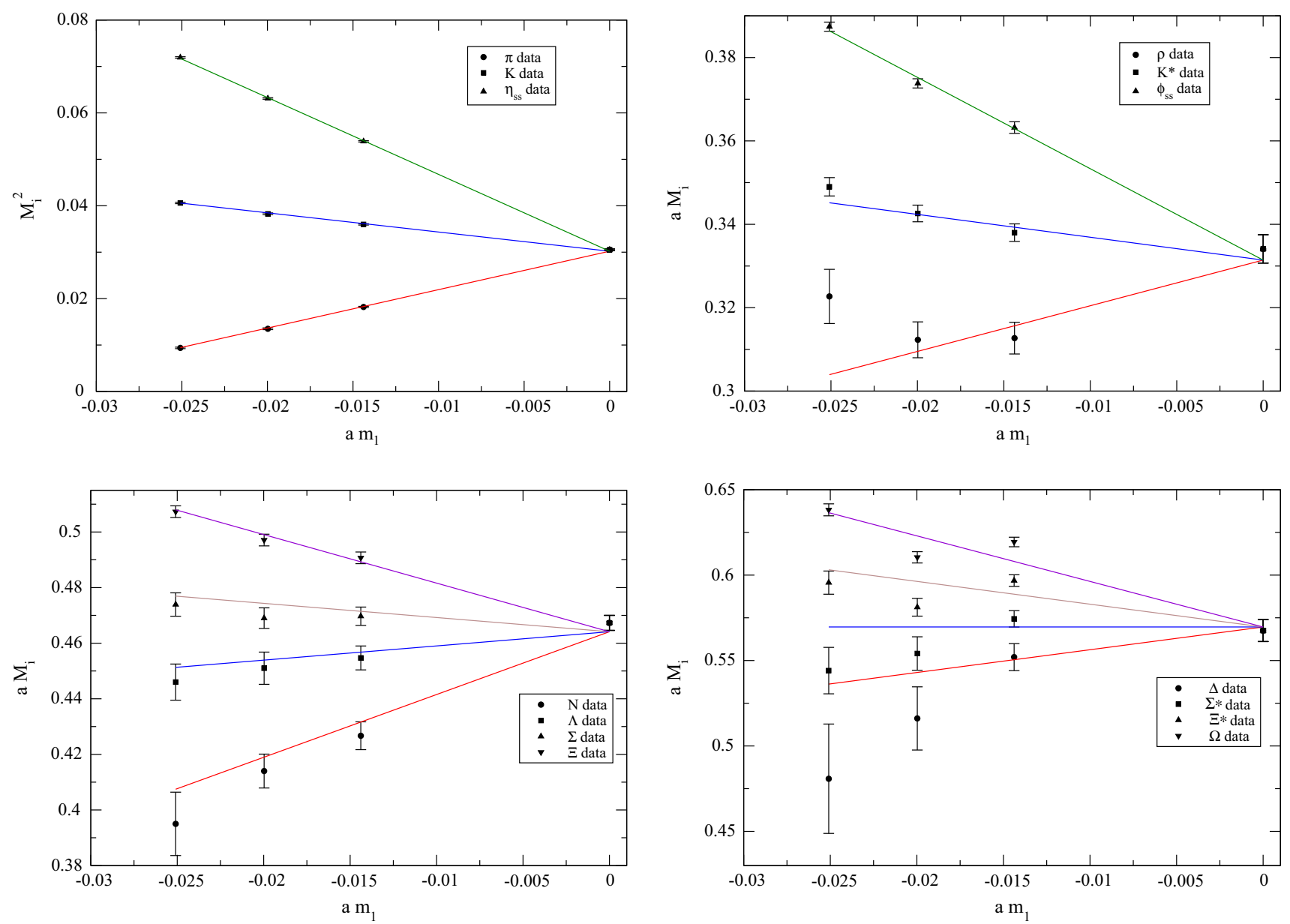

Fig. 2 Fit results for pseudoscalar (left up panel) and vector (right up panel) mesons and octet (left down panel) and decuplet (right down panel) baryons along the non-symmetric line

$\frac{2 \alpha_{P}}{\lambda_{P}}=1$

In the case of the vector mesons as well as for the octet and decuplet baryons, using the results in Eqs. (47) and (49), together with the renormalization constant in (50), one gets for the ratios in Eq. (41) the values:

Vector mesons:

$\frac{2 \alpha_{V}}{\lambda_{V}}=0.778 \pm 0.014$

Baryon octet:

$\frac{3 A_{1}}{\lambda_{B}}=0.635 \pm 0.080, \quad \frac{3 A_{2}}{\lambda_{B}}=0.144 \pm 0.023$,

Baryon decuplet:

$\frac{3 A}{\lambda_{D}}=0.590 \pm 0.202$
These results confirm a behavior for vectors and octet and decuplet baryons close to the quark model prediction, with deviations compatible with a $1 / N_{c} \simeq 30 \%$ correction, as expected from our analysis in Sect. 3. In particular, the results for vector mesons are far away from a tetraquark prediction, ${ }^{2}$ confirming their ordinary $\bar{q} q$ nature, as it has been known for long from vector meson dominance models $[56,57]$ or the $1 / N_{c}$ expansion [58-63]. These results are hence a check of the consistency of our method, opening the way to its application to non-ordinary meson candidates, as for instance the light scalar mesons. Nevertheless, this requires a generalization of the Feynman-Hellman theorem for resonances. That is what we will address in the next Section.

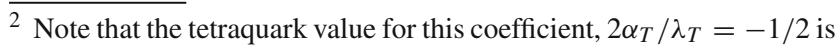
clearly different from the corresponding quark-model value of 1 , even taking into account the corrections of order of $30 \%$, which are attributed to the $O\left(N_{c}^{-1}\right)$ effects. This verifies the predictive power of the proposed criterion.
} 


\section{Unstable particles}

In this section, we present a derivation of the analog of the Feynman-Hellmann theorem for resonances in quantum field theory. To this end, we first consider stable (w.r.t. the strong interactions) particles again (say, the Goldstone bosons) and give a derivation in the language of the Green functions, which does not refer to the eigenstates and eigenvalues of the QCD Hamiltonian at all.

Let $P^{a}(x)=\bar{q}(x) i \gamma_{5} \frac{1}{2} \lambda^{a} q(x)$ be a composite field that describes the Goldstone bosons. Here, $\lambda^{a}, a=1, \ldots 8$, are the Gell-Mann flavor matrices. The two-point function of these fields

$D^{a b}\left(p^{2}\right)=i \int d^{4} x e^{i p x}\left\langle 0\left|T P^{a}(x) P^{b}(0)\right| 0\right\rangle$

contains a single pole at the physical Goldstone boson mass

$D^{a b}\left(p^{2}\right) \rightarrow \frac{\delta^{a b} Z_{a}}{M_{a}^{2}-p^{2}}+\cdots, \quad a=1, \ldots 8$.

Here, the ellipses stand for the terms which are regular at $p^{2} \rightarrow M_{a}^{2}$. For simplicity, we do not consider the case $a=$ 8 any further, as $\eta-\eta^{\prime}$ mixing would have to be taken into account. The constant $Z_{a}$ can be expressed via the matrix element of $P^{a}$ between the vacuum and the one-particle state:

$Z_{a}^{1 / 2}=\left\langle 0\left|P^{a}(0)\right| P, a\right\rangle$.

Next, we shall use the fact that, in perturbation theory, one can shuffle the quark mass term between the free Lagrangian and the interaction part. We shall use this freedom to put all of the mass term into the interaction. ${ }^{3}$ In this case, the two-point function is given by

$$
\begin{aligned}
\left\langle 0\left|T P^{a}(x) P^{b}(0)\right| 0\right\rangle= & U_{0}^{-1}\left\langle\Omega_{0}\right| T P_{0}^{a}(x) P_{0}^{b}(0) \\
& \times \exp \left(i \int d^{4} y \mathcal{L}_{\text {int }}(y)\right)\left|\Omega_{0}\right\rangle, \\
U_{0}= & \left\langle\Omega_{0}\right| T \exp \left(i \int d^{4} y \mathcal{L}_{\text {int }}(y)\left|\Omega_{0}\right\rangle,\right.
\end{aligned}
$$

where the interaction part of the QCD Lagrangian is split into the quark-mass-dependent and quark-mass-independent parts according to

$\mathcal{L}_{\text {int }}(x)=\mathcal{L}_{0}(x)-\sum_{q} Z_{F}^{-1} Z_{m} m_{q}^{r} \bar{q}_{0}(x) q_{0}(x)$.

Here, $q_{0}(x)$ is the free massless quark field, $P_{0}^{a}(x)=$ $\bar{q}_{0}(x) i \gamma_{5} \frac{1}{2} \lambda^{a} q_{0}(x)$, and $\left|\Omega_{0}\right\rangle$ denotes the vacuum in the theory with massless quarks. The quark-mass-independent part of the Lagrangian is not shown explicitly. Further, $Z_{F}, Z_{m}$

\footnotetext{
3 A mass-independent renormalization scheme is used throughout the article.
}

denote the quark wave function and quark mass renormalization constants, respectively, and $m_{q}^{r}$ are the renormalized quark masses. For simplicity, the minimal subtraction scheme is implied, where $Z_{F}, Z_{m}$ do not depend on the quark flavor and masses.

Let us now ask the question: how do the Goldstone boson masses depend on the renormalized quark masses $m_{q}^{r}(\mu)$, with the scale $\mu$ and the renormalized coupling constant $g^{r}(\mu)$ fixed? In order to answer this question, we shall first differentiate Eq. (56) with respect to the quark mass $m_{q}^{r}$ :

$$
\frac{\partial D^{a b}\left(p^{2}\right)}{\partial m_{q}^{r}} \rightarrow-\frac{Z_{a} \delta^{a b}}{\left(M_{a}^{2}-p^{2}\right)^{2}} \frac{\partial M_{a}^{2}}{\partial m_{q}^{r}}+\text { single pole }+ \text { regular. }
$$

On the other hand, differentiating Eq. (58) with respect to $m_{q}^{r}$, one obtains

$$
\begin{aligned}
& \frac{\partial D^{a b}\left(p^{2}\right)}{\partial m_{q}^{r}}=\int \mathrm{d}^{4} x e^{i p x}\left\{U_{0}^{-1}\left\langle\Omega_{0}\right| T P_{0}^{a}(x) P_{0}^{b}(0)\right. \\
& \quad \times \exp \left(i \int \mathrm{d}^{4} y \mathcal{L}_{\text {int }}(y)\right) \\
& \quad \times \int \mathrm{d}^{4} z Z_{F}^{-1} Z_{m} \bar{q}_{0}(z) q_{0}(z)\left|\Omega_{0}\right\rangle \\
& \quad-U_{0}^{-2} \frac{\partial U_{0}}{\partial m_{q}^{r}}\left\langle\Omega_{0}\right| T P_{0}^{a}(x) P_{0}^{b}(0) \\
& \left.\quad \times \exp \left(i \int \mathrm{d}^{4} y \mathcal{L}_{\text {int }}(y)\right)\left|\Omega_{0}\right\rangle\right\} \\
& =\int \mathrm{d}^{4} x \mathrm{~d}^{4} z e^{i p(x-z)}\left\{U_{0}^{-1}\left\langle\Omega_{0}\right| T P_{0}^{a}(x) P_{0}^{b}(z)\right. \\
& \quad \times \exp \left(i \int \mathrm{d}^{4} y \mathcal{L}_{\text {int }}(y)\right) \\
& \quad \times Z_{F}^{-1} Z_{m} S_{0}(0)\left|\Omega_{0}\right\rangle \\
& \quad-U_{0}^{-1}\left\langle\Omega_{0}\left|T P_{0}^{a}(x) P_{0}^{b}(z) \exp \left(i \int \mathrm{d}^{4} y \mathcal{L}_{\text {int }}(y)\right)\right| \Omega_{0}\right\rangle \\
& \left.\quad \times U_{0}^{-1}\left\langle\Omega_{0}\left|T Z_{F}^{-1} Z_{m} S_{0}(0) \exp \left(i \int \mathrm{d}^{4} y \mathcal{L}_{\text {int }}(y)\right)\right| \Omega_{0}\right\rangle\right\} .
\end{aligned}
$$

Here, $S_{0}(x)=\bar{q}_{0}(x) q_{0}(x)$ is the (unrenormalized) scalar density. It is now seen that the above equation can be written in the form

$$
\frac{\partial D^{a b}\left(p^{2}\right)}{\partial m_{q}^{r}}=\Gamma^{a b}(p, p),
$$

where

$$
\begin{gathered}
\Gamma^{a b}(p, q)=\int d^{4} x d^{4} z e^{i p x-i q z}\left\langle 0\left|T P^{a}(x) P^{b}(z) S^{r}(0)\right| 0\right\rangle_{\mathrm{conn}}, \\
\left\langle 0\left|T P^{a}(x) P^{b}(z) S^{r}(0)\right| 0\right\rangle_{\mathrm{conn}}=\left\langle 0\left|T P^{a}(x) P^{b}(z) S^{r}(0)\right| 0\right\rangle \\
-\left\langle 0\left|T P^{a}(x) P^{b}(z)\right| 0\right\rangle\left\langle 0\left|T S^{r}(0)\right| 0\right\rangle,
\end{gathered}
$$


and $S^{r}(x)=Z_{F}^{-1} Z_{m} S_{0}(x)$ denotes the renormalized scalar density.

Further, inserting a complete set of states in the above equation, it is straightforward to see that the quantity $\Gamma^{a b}(p, q)$ contains a double pole in the variables $p^{2}, q^{2}$ :

$$
\begin{aligned}
& \Gamma^{a b}(p, q) \rightarrow \\
& \quad-\frac{\delta^{a b}\left\langle 0\left|P^{a}(0)\right| P, a\right\rangle\left\langle P, a\left|S^{r}(0)\right| P, b\right\rangle\left\langle P, b\left|P^{b}(0)\right| 0\right\rangle}{\left(M_{a}^{2}-p^{2}\right)\left(M_{a}^{2}-q^{2}\right)}+\cdots,
\end{aligned}
$$

where the ellipses denote the less singular terms. Comparing the coefficients in front of the double pole in Eqs. (60) and (64), we finally arrive at the Feynman-Hellmann theorem,

$\frac{\partial M_{a}^{2}}{\partial m_{q}^{r}}=\left\langle P, a\left|S^{r}(0)\right| P, a\right\rangle$.

As already mentioned, the advantage of this derivation as compared to the standard one is that it does not refer to the (real) eigenvalues of the Hamiltonian $E_{n}(\lambda)$ from the beginning, dealing instead with the Green functions in QCD. Hence, this derivation can be directly generalized to the resonances. ${ }^{4}$ In the latter case, the two-point function is defined by an expression similar to Eq. (55) where, instead of $P^{a}(x)$, one may use any operator with the quantum number of a given resonance. Then the two-point function has a pole on some unphysical Riemann sheet of the complex $p^{2}$-plane and not on the real axis. By the same token, the matrix element of the operator $S^{r}(x)$ between the resonance "states" is defined similarly to Eq. (64), through the residue of the three-point function at the double pole. ${ }^{5}$ Consequently, the only difference between the stable states and the resonances boils down to the question whether a pole is real or not. This difference is inessential for the derivation of the Feynman-Hellmann theorem, which is given above. Consequently, it still holds, if one interprets $M_{a}^{2}$ as a resonance pole position in the complex plane and not as the energy of an isolated energy level on the

\footnotetext{
${ }^{4}$ In the above derivation, one might feel slightly uncomfortable with the trick that introduces massless quarks at the intermediate stage of the proof, albeit no reference to these is left in the final expression. This (superficial) problem can, however, easily be avoided. Instead of putting whole quark mass term into the perturbation, one could, e.g., introduce the formal parameters $s_{q}=m_{q}-m_{q}^{\text {phys }}$. The derivative in the Feynman-Hellmann theorem is taken with respect to the parameters $s_{q}$ at $s_{q}=0$, and one arrives at the same result at the end. In order to keep the notations as simple as possible, we refrained from introducing the additional parameters $s_{q}$.

5 These quantities in principle can be extracted from the lattice data, see [64-66], at least for well-isolated narrow resonances, which decay into the two-particle final states, albeit the presence of multiple decay channels renders the extraction a rather complicated exercise. Further, as an example of a calculation of the resonance matrix element in ChPT, we refer to [67]. The quark mass dependence of the $\sigma$-meson pole in the unitarized ChPT has been addressed in Ref. [68].
}

lattice. Furthermore, the large- $N_{c}$ and SU(3) symmetry arguments apply to the Green functions irrespective of whether they have a real or a complex pole. Consequently, the physical meaning of the parameters $\gamma, \gamma^{\prime}, \beta, \beta^{\prime}$ remains the same, albeit they become complex for resonances. Namely, if these happen to be close to the (real) quark model values for a given multiplet, then this multiplet has a little admixture of exotica and vice versa.

One may also wonder whether the Gell-Mann-Okubo formula, which was extensively used above, is applicable in the case of the resonances. The answer to this question can be found along a similar pattern. The quark mass term in the Lagrangian can be rewritten as (to ease notations, the renormalization constants are suppressed)

$$
\begin{aligned}
\mathcal{L}_{m}= & \hat{m}(\bar{u} u+\bar{d} d)+m_{s} \bar{s} s=\bar{m}(\bar{u} u+\bar{d} d+\bar{s} s) \\
& +\lambda \frac{m_{1}}{3}(\bar{u} u+\bar{d} d-2 \bar{s} s),
\end{aligned}
$$

where $\lambda=1$ in the real world. Suppose now that, for any $\lambda$, one has a multiplet of poles $M_{a}^{2}$ (real or complex) in the two-point function of the operators with appropriate quantum numbers. One could now differentiate with respect to the parameter $\lambda$ and get

$\frac{\partial M_{a}^{2}}{\partial \lambda}=\left\langle P, a\left|O_{8}^{r}(0)\right| P, a\right\rangle$,

where $O_{8}^{r}$ denotes the renormalized operator proportional to $\bar{u} u+\bar{d} d-2 \bar{s} s$. In the case of the resonances, the matrix element in the right-hand side is understood, as the residue of the pertinent three-point function at the complex pole.

The above relation is written for any $\lambda$. For $\lambda=1$, we are back to the real world. One may first consider it for $\lambda \rightarrow 0$, where it yields the first-order correction to the $\mathrm{SU}(3)$ symmetric limit. The Lagrangian is explicitly SU(3) symmetric, as $\lambda \rightarrow 0$, and both the interpolating particle fields and the operator $O_{8}^{r}$ transform as irreducible tensor operators of SU(3). The group-theoretical analysis applies directly to the three-point function, and the Gell-Mann-Okubo formula holds-even for the resonances.

Continuation to $\lambda=1$, i.e., back to the real world, is a subtle issue. The (approximate) validity of the Gell-MannOkubo formula is, in fact, equivalent to the statement that the linear term in $\lambda$ describes the spectrum well up to $\lambda=1$. In case of stable particles, there exists no internal contradiction in assuming this. Consider, however, the situation, when all particles in the multiplet are stable at the SU(3)-symmetry point. Increasing $\lambda$ introduces the mass splitting, and some of the particles become unstable at $\lambda=\lambda_{\text {crit }}<1$. Assuming the analyticity in $\lambda$ then leads to the controversy since, as it is well known, the real and imaginary parts of the pole position have cusps at threshold. Consequently, the assumption that the Gell-Mann-Okubo formula approximately holds for the resonance masses as well implies that the cusp effects are 
small, and hence this approximation should work better for the resonances with higher spin.

Finally, a few words about testing the exotic content of the resonances with the use of the lattice simulations. As is well known, the resonances do not correspond to the individual energy levels of the lattice QCD spectrum. In order to extract the position of the resonance pole, one has to first determine the phase shift at a given energy by use of the Lüscher equation and, at the next stage, find the pole position through the extrapolation into the complex energy plane. Recent years have seen some progress in this direction; see, e.g., Refs. [69,70], which is summarized in the recent review in Ref. [71]. More work is, however, necessary to perform a full-fledged investigation of exotic resonances on the lattice.

\section{Conclusions}

1. We propose a criterion which allows one to judge whether the hadrons in a given multiplet are predominately quark-model states or exotic states. The quantities $\gamma, \gamma^{\prime}, \beta, \beta^{\prime}$ for different multiplets are observable quantities, expressed through the $\sigma$-terms. In the quark model, these quantities are exactly given by the grouptheoretical factors. Should it turn out that the values of these quantities for some multiplet in the real world significantly differ from the quark model values, then one would interpret this as a signature of the exotic character of a multiplet in question.

2. The above criterion has been verified, using Chiral Perturbation Theory and large- $N_{c}$ arguments. It has been shown that the quark model values for $\gamma, \gamma^{\prime}, \beta, \beta^{\prime}$ emerge in QCD at the leading order in $1 / N_{c}$, both in the meson and in the baryon sector.

3. Using the hadron mass values, measured at different input values of the quark masses in lattice QCD simulations, we have verified our criterion in the case of the pseudoscalar and vector meson octets, as well as the lowlying baryon octet and decuplet. As expected, the fit to the lattice data gives results close to the quark model predictions. In some parameters, the difference of order of $1 / N_{c} \simeq 30 \%$ is observed. It will be extremely important to apply the same criterion to the lightest scalar meson octet, which is the most obvious candidate for the lowlying exotic multiplet.

4. The Feynman-Hellmann theorem, which has been used above to calculate the quantities $\gamma, \gamma^{\prime}, \beta, \beta^{\prime}$, has been generalized for the resonance states. A field-theoretical proof is provided. The criterion for the exotic multiplets does not change its form. Such a generalization is necessary, because all candidates for QCD exotica are resonances and not stable particles.
Acknowledgements The authors thank J. M. Alarcon, M. Frink, J. Gasser, H. Leutwyler, F. J. Llanes-Estrada, J. Nebreda, A. Manohar and J. R. Pelaez for useful discussions. GS thanks his colleagues of the QCDSF collaboration for sharing the lattice results. We acknowledge the support from the DFG (CRC 110 "Symmetries and the Emergence of Structure in QCD", Grant No. TRR110). This research is supported in part by Volkswagenstiftung under Contract no. 86260, by the Chinese Academy of Sciences (CAS) President's International Fellowship Initiative (PIFI) (Grant no. 2017VMA0025), the Swiss National Science Foundation (SNF), and by Shota Rustaveli National Science Foundation (SRNSF), Grant no. DI-2016-26.

Open Access This article is distributed under the terms of the Creative Commons Attribution 4.0 International License (http://creativecomm ons.org/licenses/by/4.0/), which permits unrestricted use, distribution, and reproduction in any medium, provided you give appropriate credit to the original author(s) and the source, provide a link to the Creative Commons license, and indicate if changes were made.

Funded by $\mathrm{SCOAP}^{3}$.

\section{References}

1. H. Hellmann, Einführung in die Quantenchemie (Deuticke, Leipzig und Wien, 1937)

2. R.P. Feynman, Phys. Rev. 56, 340 (1939)

3. A. Bottino, F. Donato, N. Fornengo, S. Scopel, Astropart. Phys. 13, 215 (2000). arXiv:hep-ph/9909228

4. A. Bottino, F. Donato, N. Fornengo, S. Scopel, Astropart. Phys. 18, 205 (2002). arXiv:hep-ph/0111229

5. J.R. Ellis, K.A. Olive, C. Savage, Phys. Rev. D 77, 065026 (2008). arXiv:0801.3656 [hep-ph]

6. G. Belanger, F. Boudjema, A. Pukhov, A. Semenov, Comput. Phys. Commun. 180, 747 (2009). arXiv:0803.2360 [hep-ph]

7. G. Belanger, F. Boudjema, A. Pukhov, A. Semenov, Comput. Phys. Commun. 185, 960 (2014). arXiv:1305.0237 [hep-ph]

8. A. Crivellin, M. Hoferichter, M. Procura, L.C. Tunstall, JHEP 1507, 129 (2015). arXiv: 1503.03478 [hep-ph]

9. M. Hoferichter, P. Klos, A. Schwenk, Phys. Lett. B 746, 410 (2015). arXiv:1503.04811 [hep-ph]

10. V. Cirigliano, R. Kitano, Y. Okada, P. Tuzon, Phys. Rev. D 80 , 013002 (2009). arXiv:0904.0957 [hep-ph]

11. A. Crivellin, M. Hoferichter, M. Procura, Phys. Rev. D 89, 093024 (2014). arXiv:1404.7134 [hep-ph]

12. R.J. Crewther, P. Di Vecchia, G. Veneziano, E. Witten, Phys. Lett. 88B, 123 (1979). Erratum: [Phys. Lett. 91B, 487 (1980)]

13. J. Bsaisou, C. Hanhart, S. Liebig, U.-G. Meißner, A. Nogga, A. Wirzba, Eur. Phys. J. A 49, 31 (2013). arXiv:1209.6306 [hep-ph]

14. J. Engel, M.J. Ramsey-Musolf, U. van Kolck, Prog. Part. Nucl. Phys. 71, 21 (2013). arXiv:1303.2371 [nucl-th]

15. J. de Vries, U.-G. Meißner, Int. J. Mod. Phys. E 25(05), 1641008 (2016). arXiv:1509.07331 [hep-ph]

16. J. de Vries, E. Mereghetti, A. Walker-Loud, Phys. Rev. C 92(4), 045201 (2015). arXiv:1506.06247 [nucl-th]

17. N. Yamanaka, B.K. Sahoo, N. Yoshinaga, T. Sato, K. Asahi, B.P. Das, Eur. Phys. J. A 53, 54 (2017). arXiv:1703.01570 [hep-ph]

18. D.B. Kaplan, A.E. Nelson, Phys. Lett. B 175, 57 (1986)

19. A.E. Nelson, D.B. Kaplan, Phys. Lett. B 192, 193 (1987)

20. U.-G. Meißner, J.A. Oller, A. Wirzba, Annals Phys. 297, 27 (2002). arXiv:nucl-th/0109026

21. J. Gasser, H. Leutwyler, M.E. Sainio, Phys. Lett. B 253, 252 (1991)

22. M. Hoferichter, J. Ruiz de Elvira, B. Kubis, U.-G. Meißner, Phys. Rev. Lett. 115, 092301 (2015). arXiv:1506.04142 [hep-ph]

23. M. Hoferichter, J. Ruiz de Elvira, B. Kubis, U.-G. Meißner, Phys. Rep. 625, 1 (2016). arXiv:1510.06039 [hep-ph] 
24. J. Ruiz de Elvira, M. Hoferichter, B. Kubis, U.-G. Meißner, arXiv:1706.01465 [hep-ph]

25. R. Horsley et al. [QCDSF-UKQCD Collaboration], Phys. Rev. D 85, 034506 (2012). arXiv:1110.4971 [hep-lat]

26. P. Junnarkar, A. Walker-Loud, Phys. Rev. D 87, 114510 (2013). arXiv:1301.1114 [hep-lat]

27. S. Durr et al., Phys. Rev. Lett. 116(17), 172001 (2016). arXiv: 1510.08013 [hep-lat]

28. Y.B. Yang et al. [xQCD Collaboration], Phys. Rev. D 94(5), 054503 (2016). arXiv:1511.09089 [hep-lat]

29. A. Abdel-Rehim et al. [ETM Collaboration], Phys. Rev. Lett. 116(25), 252001 (2016). arXiv:1601.01624 [hep-lat]

30. G.S. Bali et al., Nucl. Phys. B 866, 1 (2013). arXiv:1206.7034 [hep-lat]

31. G.S. Bali et al. [RQCD Collaboration], Phys. Rev. D 93(9), 094504 (2016). arXiv:1603.00827 [hep-lat]

32. M. Hoferichter, J. Ruiz de Elvira, B. Kubis, U.-G. Meißner, Phys. Lett. B 760, 74 (2016). arXiv:1602.07688 [hep-lat]

33. V. Bernard, M. Lage, U.-G. Meißner, A. Rusetsky, JHEP 1101, 019 (2011). arXiv:1010.6018 [hep-lat]

34. P. Ziesche, K. Kunze, B. Milek, J. Phys. A 20, 2859 (1987)

35. A. Tachibana, T. Yamabe, K. Fukui, J. Phys. B 10, 3175 (1977)

36. G.'t Hooft, G. Isidori, L. Maiani, A.D. Polosa, Phys. Lett 662, 424 (2008). arXiv:0801.2288 [hep-ph]

37. T.P. Cheng, Phys. Rev. D 13, 2161 (1976)

38. E. Witten, Nucl. Phys. B 160, 57 (1979)

39. G. Karl, J.E. Paton, Phys. Rev. D 30, 238 (1984)

40. A.J. Buchmann, R.F. Lebed, Phys. Rev. D 62, 096005 (2000). arXiv:hep-ph/0003167

41. R.F. Lebed, Czech. J. Phys. 49, 1273 (1999). arXiv:nucl-th/9810080

42. R.F. Dashen, E.E. Jenkins, A.V. Manohar, Phys. Rev. D 49, 4713 (1994). Erratum: [Phys. Rev. D 51, 2489 (1995)]. arXiv:hep-ph/9310379

43. R.F. Dashen, E.E. Jenkins, A.V. Manohar, Phys. Rev. D 51, 3697 (1995). arXiv:hep-ph/9411234

44. E.E. Jenkins, Phys. Rev. D 53, 2625 (1996). arXiv:hep-ph/9509433

45. J. Gasser, H. Leutwyler, Nucl. Phys. B 250, 465 (1985)

46. J. Bijnens, G. Ecker, Ann. Rev. Nucl. Part. Sci. 64, 149 (2014). arXiv:1405.6488 [hep-ph]

47. V. Bernard, N. Kaiser, U.-G. Meißner, Z. Phys. C 60, 111 (1993). arXiv:hep-ph/9303311

48. B. Borasoy, U.-G. Meißner, Ann. Phys. 254, 192 (1997). arXiv:hep-ph/9607432

49. M. Frink, U.-G. Meißner, JHEP 0407, 028 (2004). arXiv:hep-lat/0404018
50. M. Hoferichter, J. Ruiz de Elvira, B. Kubis, U.-G. Meißner, Phys. Rev. Lett. 115(19), 192301 (2015). arXiv:1507.07552 [nucl-th]

51. D. Siemens, J. Ruiz de Elvira, E. Epelbaum, M. Hoferichter, H. Krebs, B. Kubis, U.-G. Meißner, Phys. Lett. B 770, 27 (2017). arXiv: 1610.08978 [nucl-th]

52. X.-L. Ren, L.S. Geng, J. Martin Camalich, J. Meng, H. Toki, JHEP 1212, 073 (2012). arXiv:1209.3641 [nucl-th]

53. X.L. Ren, L.S. Geng, J. Meng, PoS CD 15, 085 (2016) arXiv:1512.08841 [nucl-th]

54. J. Bijnens, P. Gosdzinsky, P. Talavera, Nucl. Phys. B 501, 495 (1997). arXiv:hep-ph/9704212

55. W. Bietenholz et al., Phys. Rev. D 84, 054509 (2011). arXiv:1102.5300 [hep-lat]

56. J. Gasser, H. Leutwyler, Ann. Phys. 158, 142 (1984)

57. G. Ecker, J. Gasser, A. Pich, E. de Rafael, Nucl. Phys. B 321, 311 (1989)

58. J.R. Pelaez, Phys. Rev. Lett. 92, 102001 (2004). arXiv:hep-ph/0309292

59. J. Ruiz de Elvira, J.R. Pelaez, M.R. Pennington, D.J. Wilson, Phys. Rev. D 84, 096006 (2011). arXiv:1009.6204 [hep-ph]

60. Z.H. Guo, J.A. Oller, J. Ruiz de Elvira, Phys. Lett. B 712, 407 (2012). arXiv:1203.4381 [hep-ph]

61. Z.H. Guo, J.A. Oller, J. Ruiz de Elvira, Phys. Rev. D 86, 054006 (2012). arXiv:1206.4163 [hep-ph]

62. T. Cohen, F.J. Llanes-Estrada, J.R. Pelaez, J. Ruiz de Elvira, Phys. Rev. D 90(3), 036003 (2014). arXiv:1405.4831 [hep-ph]

63. T. Ledwig, J. Nieves, A. Pich, E. Ruiz Arriola, J. Ruiz de Elvira. Phys. Rev. D 90(11), 114020 (2014). arXiv:1407.3750 [hep-ph]

64. V. Bernard, D. Hoja, U.-G. Meißner, A. Rusetsky, JHEP 1209, 023 (2012). arXiv:1205.4642 [hep-lat]

65. A. Agadjanov, V. Bernard, U.-G. Meißner, A. Rusetsky, Nucl. Phys. B 886, 1199 (2014). arXiv:1405.3476 [hep-lat]

66. A. Agadjanov, V. Bernard, U.-G. Meißner, A. Rusetsky, Nucl. Phys. B 910, 387 (2016). arXiv:1605.03386 [hep-lat]

67. M. Albaladejo, J.A. Oller, Phys. Rev. D 86, 034003 (2012). arXiv:1205.6606 [hep-ph]

68. P.C. Bruns, arXiv:1610.00119 [nucl-th]

69. R.A. Briceno, J.J. Dudek, R.G. Edwards, D.J. Wilson, Phys. Rev. Lett. 118(2), 022002 (2017). arXiv:1607.05900 [hep-ph]

70. D.J. Wilson, R.A. Briceno, J.J. Dudek, R.G. Edwards, C.E. Thomas, Phys. Rev. D 92(9), 094502 (2015). arXiv:1507.02599 [hep-ph]

71. R.A. Briceno, J.J. Dudek, R.D. Young, arXiv:1706.06223 [hep-lat] 